\title{
Design and Properties of Magnetically Controlled Sorbents
}

\author{
O.A. Butusova \\ Moscow Aviation Institute (National Research University), Volokolamskoe shosse, 4, 125993, Moscow, Russia \\ gxtl@mail.ru
}

Article History: Received: 11 January 2021; Accepted: 27 February 2021; Published online: 5 April 2021

\begin{abstract}
In this work, the main parameters and advantages of magnetically controlled sorbents are considered. A large surface of nanodispersed magnetically controlled sorbents adsorbs large amounts of toxins, therefore, the consumption of magnetically controlled sorbents in each operation is insignificant. The ability to quickly replace the spent magnetically controlled sorbents in the detoxification apparatus with a new portion of magnetically controlled sorbents increases the reliability, simplicity and ease of use of the method under consideration. The small thickness of the sorbent layer and the availability of the sorbent surface provide almost instantaneous establishment of equilibrium concentrations, which significantly reduces the required processing time for the biofluid and increases the productivity of the purification process.
\end{abstract}

Keywords: Magnetically controlled sorbents, adsorption, toxins, biofluids.

\section{Introduction}

One of the modern achievements of nanotechnology is the creation of nano- and micro-sized composite materials and technologies for their industrial production [1-13]. Among these materials, powders of magnetically controlled sorbents play important role in medicine. The name itself defines the most important property of magnetically controlled sorbents - the ability to remotely control them using an external magnetic field. The combination of this property with the possibility of drug loading and those biochemical properties that can be endowed with particles with the help of a suitable coating make it possible to create fundamentally new methods of treating various diseases. Therefore, the issues related to the use of magnetically controlled sorbents in general medical practice are relevant. In particular, one of these issues is the development of a methodology for the practical calculation of the parameters of the extracorporeal detoxification system, which is considered in this work.

\section{Methods of using magnetically controlled sorbents in medicine}

Coatings on nano and micro-sized particles can serve for many purposes. First of all, modification of the surface with coatings makes it possible to make the particles compatible with various matrixes [14-20]. For medical purposes, the biocompatibility with the environments of a living organism is of crucial importance. It is equally important that coatings can significantly enhance or decrease the sorption properties of magnetically controlled sorbents. This provides prerequisites for the creation of magnetically controlled particles with specific sorption properties. It is also known that the coatings prevent the core from leaching out. The presence of a coating also often facilitates the stabilization of particles in an environment with an alkaline $\mathrm{pH}$ or significant salt concentration. For example, the isoelectric point of $\mathrm{SiO}_{2}$ is reached at $\mathrm{pH}$ 2-3. Therefore, the particles coated with silica are negatively charged at the $\mathrm{pH}$ of the blood, which causes electrostatic repulsion, which avoids the formation of clumps.

The unique physicochemical properties of magnetically controlled sorbents, noted above and many others, attract great interest of researchers of various specialties around the world. This is evidenced by the growth in the number of scientific publications, patents and innovative works related to both the study of the fundamental properties of magnetically controlled particles and the solution of applied problems associated with the development of methods for their targeted use in medicine and biology.

This work is a continuation of previous works, which presents the results of comparative studies of the structure and sorption capacity of nano- and micro-sized particles to low-, medium- and high-molecular objects [21-27].

In addition to being used in devices for the standardized extraction of reagents in biofluid, magnetically controlled sorbents can find no less effective use for introducing useful substances (vitamins, microelements, or other additives) into biofluid. Indeed, the release of the active reagent from the shallow pores of the specified sorbent (a thin layer of the carbon shell) is facilitated, does not require much time and is easily controlled. All this makes the specified process well predictable. Accordingly, the rate of establishment of the equilibrium concentration of the active reagent introduced into the biofluid is high, and the current value of the reagent concentration in the solution is quite accurately determined by the surface density of the reagent and the thermodynamic characteristics of the system, taking into account the Langmuir isotherm, and can be purposefully changed depending on the solution temperature.

\section{Principles of operation of magnetically controlled sorbents}

Taking into account the focus of this work on substantiating the method of practical calculation of the parameters of the extracorporeal detoxification system, it seems appropriate to determine the dependence of the concentration of the adsorbed component in the solution on the characteristics of the type of magnetically controlled sorbents used (mass, sorp- 
tion capacity). Obtaining quantitative estimates will make it possible to reasonably set the required dosage of magnetically controlled sorbents for a specific detoxification procedure. Important, that temperature conditions can strongly affect the material and its behavior, so, a range of works was devoted to modelling of temperature conditions of materials [28-38]. To achieve the task, it is useful to consider the essence of the used physical phenomenon - adsorption and clarify the meaning of the characteristic parameters describing it.

The adsorption capacity of any adsorbent is determined primarily by its specific surface area, $s_{0}=s / m$ where $s_{0}$ is the surface area of the adsorbent; $m=V d$ - its mass; $\mathrm{d}\left(\mathrm{kg} / \mathrm{m}^{3}\right)$ - the density of the adsorbent, $\mathrm{V}\left(\mathrm{m}^{3}\right)$ - the volume. From this we obtain $s_{0}=s /(V d)=(1 / d) \delta$, where $\delta=s / V$ is the degree of dispersion (fragmentation) of the adsorbent.

Specific surface area of the adsorbent $S_{0}$, and, consequently, its adsorption capacity will be the greater, the greater its degree of dispersion $\delta$, or the smaller the linear dimensions of the particles into which the adsorbent is crushed. Active (well absorbing) adsorbents have a very large specific surface area. For example, the absorbers most often used for scientific purposes and industrial practice - activated carbon, silica gel, zeolites - have $S_{0}$ up to several hundred and even thousands of square meters per $1 \mathrm{~g}$.

The ability of the adsorbent to absorb the adsorbate is characterized by the amount of adsorption. The amount of adsorption is the excess mass of the adsorbate in the boundary layer over its mass in an equal volume of the environment, referred to the unit surface of the adsorbent.

Sometimes the adsorption value is expressed in moles of adsorbate per $1 \mathrm{~m}^{2}$ (or $1 \mathrm{~cm}^{2}$ ) of the adsorbent surface. Since quite often the surface of the adsorbent is unknown, the value of adsorption is expressed in moles of adsorbate per $1 \mathrm{~g}$ of adsorbent ( $\mathrm{mol} / \mathrm{g}$ ). It is customary to evaluate the process of toxin sorption by the adsorbing surface using the curves of Langmuir sorption isotherms.

As you know, the surface of solids, like liquids, has an excess free Gibbs energy. However, solids, unlike liquids, cannot change the surface area by spontaneous change in shape. Therefore, the tendency to a decrease in the excess surface Gibbs energy in systems where the solid phase (the surface of magnetically controlled sorbents) is in contact with a liquid solution manifests itself mainly in the ability to retain solute molecules on the surface. The latter is possible if the molecules of the solute interact with the surface more strongly than with the molecules of the solvent - the liquid to be purified (adsorption).

The dependence of the adsorbed amount of toxin $\Gamma$ on its concentration in solution at a constant temperature is called the adsorption isotherm, a typical form of which is shown below in Fig.1.

The Langmuir adsorption isotherm in coordinates $\Gamma=\Gamma\left(C_{a}\right)$ is a hyperbola and is shown in Fig.1.

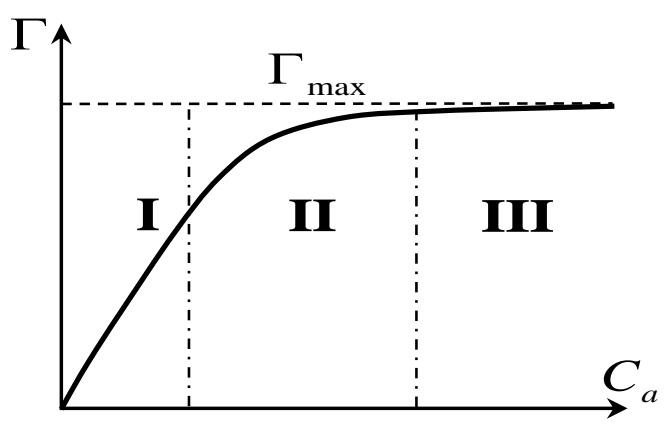

Figure: 1. Adsorption isotherm and its characteristic regions.

During adsorption, the surface of the adsorbent is gradually filled with molecules of the adsorbed substance: first, the most active areas are filled, and then the entire surface. After filling it, molecules can form a second, third, etc. layers. Therefore, a distinction is made between monomolecular and polymolecular adsorption. Given the monomolecularity of the adsorption layer, its thickness is obviously limited. In this case, the amount of toxin absorbed by the sorbent cannot exceed the limiting value $\Gamma=\Gamma_{\max }$.

Let the homogeneous surface of the adsorbent absorbing the substance be partially occupied by the adsorbate molecules. Equilibrium will be established when the number of adsorbate (toxin) molecules sorbed on the surface per unit of time becomes equal to the number of molecules leaving it during the same time. The number of sorbed molecules, expressed in mol per second, is proportional to the concentration of the adsorbate $C_{a}$ and the fraction of the surface that remains free. If the entire surface has an area of one and the occupied fraction of the surface is $\theta$, the share $(1-\theta)$ is free. It's obvious that $\theta=\Gamma / \Gamma_{\text {max }}$, i.e. $\theta$, thus, there is the degree of filling of the adsorbent with adsorbate. 
For a molecule to be adsorbed, it must hit the surface of the adsorbent and hit an unoccupied place. Due to the fact that the number of hits is proportional to the concentration of the adsorbate $C_{a}$, and the probability of hitting an unoccupied place is proportional to the fraction of the free surface $(1-\theta)$, therefore the rate of binding of molecules by the surface (adsorption rate) $V_{1}=k_{1} C_{a}(1-\theta)$, where $k_{1}$ is the rate constant of the adsorption process.

A molecule is desorbed when its energy is sufficient to detach from the surface. The number of such molecules will obviously be proportional to the total number of adsorbed molecules, i.e. $V_{2}=k_{2} \theta$, where $k_{2}$ is the rate constant of the desorption process.

Adsorption equilibrium occurs when $V_{1}=V_{2}$ or $k_{1} C_{a}(1-\theta)=k_{2} \theta$, from where:

$\frac{k_{1}}{k_{2}}=K_{p}=\frac{\theta}{C_{a}(1-\theta)}$,

where $K_{p}$ - constant of adsorption equilibrium at a given temperature.

From the last equation we find $\theta=K_{p} C_{a} /\left(1+K_{p} C_{a}\right)$. Hence, taking into account the replacement $\theta=\Gamma / \Gamma_{\max }$, get:

$\Gamma=\Gamma_{\max } K_{p} C_{a} /\left(1+K_{p} C_{a}\right)$.

This equation is the Langmuir adsorption isotherm equation for one component, where $C_{a}$ is the equilibrium concentration of the adsorbate (toxin).

For small $C_{a}$, i.e. when the relation is $1>>K_{p} C_{a}$ satisfied, we arrive at Henry's law (region I): $\Gamma=\Gamma_{\max } K_{p} C_{a}$.

In the region of high concentrations, i.e. when the relation $1<<K_{p} C_{a}$, we get equality $\Gamma=\Gamma_{\text {max }}$, corresponding to the state of saturation, when the amount of adsorbed toxin does not change with an increase in its concentration $C_{a}$ (region III).

Thus, the Langmuir equation well reflects the features of the isotherm in the region of low and in the region of high concentrations, but it does not always convey well the dependence $\Gamma=\Gamma\left(C_{a}\right)$ in the intermediate region II (Fig. 3). The latter circumstance, however, is insignificant, since when analyzing issues related to the detoxification of biofluids, we are interested in the region of low concentrations $C_{a}$, i.e. area I of the graph $\Gamma=\Gamma\left(C_{a}\right)$.

Obviously, the magnitude of adsorption depends both on the nature of the adsorbent and adsorbate, and on the conditions of the process, in particular, on the temperature of the biological environment. For example, the nature of the temperature dependence of the sorption-desorption process is shown in Fig. 2, where, for comparison, three graphs are shown corresponding to different temperatures of the process.

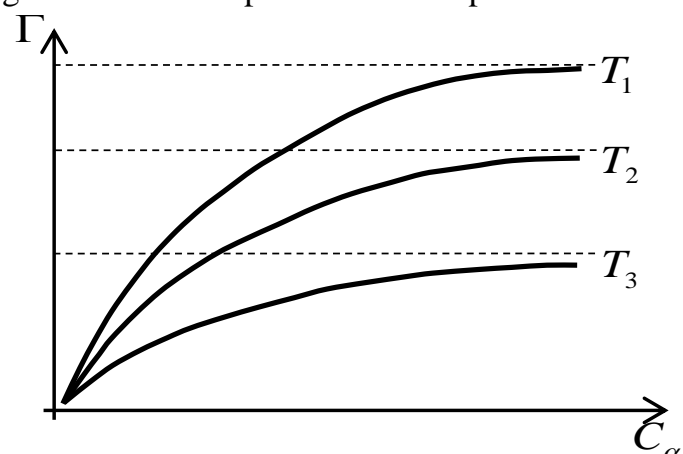

Figure: 2. Dependence of the amount of absorbed toxin on its concentration in a liquidd medium at different temperatures $T ; T_{3}>T_{2}>T_{1}$.

In order to derive the equation of the adsorption isotherm, a number of simplifications are introduced. All the places where the adsorbed particles are fixed are the same, and adsorption on one of them does not affect the state of the other. The interaction between the adsorbed particles is negligible. The adsorption layer is monomolecular, i.e. it consists of one layer of molecules. In this case, the bond of the adsorbate with the adsorbent is suf- 
ficiently strong, which excludes the movement of the adsorption complex along the surface of the adsorbent (localized adsorption).

\section{Conclusion}

The high efficiency of nanosized powders for sorption of high-molecular compounds was shown. The main regularities of the adsorption of organic compounds on the surface of solid particles are shown. The parameters of magnetically controlled sorbents are considered. The results obtained open up prospects for the use of magnetically controlled sorbents in medicine in the development of technologies and devices for extracorporeal detoxification of biological media of the body.

\section{References}

1. Rabinskiy, L.N., Sitnikov, S.A. Development of technologies for obtaining composite material based on silicone binder for its further use in space electric rocket engines// Periodico Tche Quimica, 2018, 15 (Special Issue 1), p. 390-395.

2. Formalev, V.F., Kolesnik, S.A., Kuznetsova, E.L. Identification of new law for decomposition of bonding heat-shielding composite materials/Asia Life Sciences. 2019 (1), p. 139-148.

3. Rabinskiy, L.N., Tushavina, O.V. Problems of land reclamation and heat protection of biological objects against contamination by the aviation and rocket launch site // Journal of Environmental Management and Tourism, 2019, 10(5), p. 967-973.

4. Bulychev, N.A., Rabinskiy, L.N., Tushavina, O.V. Effect of intense mechanical vibration of ultrasonic frequency on thermal unstable low-temperature plasma// Nanoscience and Technology, 2020, 11(1), p. 15-21.

5. Astapov, A.N., Kuznetsova, E.L., Rabinskiy, L.N. Operating capacity of anti-oxidizing coating in hypersonic flows of air plasma // Surface Review and Letters, 2019, 26(2), 1850145 p.

6. Rabinskiy, L.N., Tushavina, O.V., Starovoitov, E.I. Study of thermal effects of electromagnetic radiation on the environment from space rocket activity // INCAS Bulletin, 2020, 12 (Special Issue), p. 141-148.

7. Babaytsev, A.V., Orekhov, A.A., Rabinskiy, L.N. Properties and microstructure of AlSi10Mg samples obtained by selective laser melting// Nanoscience and Technology, 2020, 11(3), p. 213-222.

8. Bulychev, N.A., Bodryshev, V.V., Rabinskiy, L.N. Analysis of geometric characteristics of two-phase polymer-solvent systems during the separation of solutions according to the intensity of the image of micrographs//Periodico Tche Quimica, 2019, 16(32), p. 551-559.

9. Bulychev N. A., Kuznetsova E.L., Bodryshev V. V. Rabinskiy L.N. Nanotechnological aspects of temperature-dependent decomposition of polymer solutions, Nanoscience and Technology: An International Journal, №2, 2018, c.91-97.

10. Egorova, O.V., Kyaw, Y.K. Solution of inverse non-stationary boundary value problems of diffraction of plane pressure wave on convex surfaces based on analytical solution//Journal of Applied Engineering Science, 2020, 18(4), p. 676-680.

11. Rabinskiy, L.N., Tushavina, O.V., Formalev, V.F. Mathematical modeling of heat and mass transfer in shock layer on dimmed bodies at aerodynamic heating of aircraft// Asia Life Sciences, 2019, (2), p. 897911.

12. Antufev, B.A., Egorova, O.V., Rabinskiy, L.N. Quasi-static stability of a ribbed shell interacting with moving load// INCAS Bulletin, 2019, 11, p. 33-39.

13. Bodryshev, V.V., Babaytsev, A.V., Rabinskiy, L.N. Investigation of processes of deformation of plastic materials with the help of digital image processing// Periodico Tche Quimica, 2019, 16(33), p. 865-876.

14. B.A. Garibyan. Modelling of Technical Parameters of Discharge Reactor for Polymer Treatment, International Journal of Pharmaceutical Research, 2020, Vol. 12, Supplementary Issue 2, pp. 1833-1837.

15. A.N. Tarasova. Vibration-based Method for Mechanochemical Coating Metallic Surfaces, International Journal of Pharmaceutical Research, 2020, Vol. 12, Supplementary Issue 2, pp. 1160-1168.

16. B.A. Garibyan. Mechanical Properties of Electroconductive Ceramics, International Journal of Pharmaceutical Research, 2020, Vol. 12, Supplementary Issue 2, pp. 1825-1828.

17. M.O. Kaptakov. Effect of Ultrasonic Treatment on Stability of TiO2 Aqueous Dispersions in Presence of Water-Soluble Polymers, International Journal of Pharmaceutical Research, 2020, Vol. 12, Supplementary Issue 2, pp. 1821-1824.

18. Yu.V. Ioni. Synthesis of Metal Oxide Nanoparticles and Formation of Nanostructured Layers on Surfaces under Ultrasonic Vibrations, International Journal of Pharmaceutical Research, 2020, Vol. 12, Issue 4, pp. 3432-3435.

19. A.N. Tarasova. Effect of Reagent Concentrations on Equilibria in Water-Soluble Complexes, International Journal of Pharmaceutical Research, 2020, Vol. 12, Supplementary Issue 2, pp. 1169-1172.

20. A.N. Tarasova. Effect of Vibration on Physical Properties of Polymeric Latexes, International Journal of Pharmaceutical Research, 2020, Vol. 12, Supplementary Issue 2, pp. 1173-1180.

21. B.A. Garibyan. Enhancement of Mechanical Properties of Inorganic Glass under Ultrasonic Treatment, International Journal of Pharmaceutical Research, 2020, Vol. 12, Supplementary Issue 2, pp. 1829-1832. 
22. Yu.V. Ioni, A. Ethiraj. New Tailor-Made Polymer Stabilizers for Aqueous Dispersions of Hydrophobic Carbon Nanoparticles, International Journal of Pharmaceutical Research, 2020, Vol. 12, Issue 4, pp. 3443-3446.

23. Yu.V. Ioni. Nanoparticles of noble metals on the surface of graphene flakes, Periodico Tche Quimica, 2020, Vol. 17, No. 36, pp. 1199-1211.

24. N.A. Bulychev, A.V. Ivanov. Effect of vibration on structure and properties of polymeric membranes, International Journal of Nanotechnology, 2019, Vol. 16, Nos. 6/7/8/9/10, pp. $334-343$.

25. N.A. Bulychev, A.V. Ivanov. Nanostructure of Organic-Inorganic Composite Materials Based on Polymer Hydrogels, International Journal of Nanotechnology, 2019, Vol. 16, Nos. 6/7/8/9/10, pp. 344 - 355.

26. Yu.V. Ioni, A. Ethiraj. Study of Microparticles Surface Modification by Electrokinetic Potential Measuring, International Journal of Pharmaceutical Research, 2020, Vol. 12, Issue 4, pp. 3436-3439.

27. Yu.V. Ioni. Effect of Ultrasonic Treatment on Properties of Aqueous Dispersions of Inorganic and Organic Particles in Presence of Water-Soluble Polymers, International Journal of Pharmaceutical Research, 2020, Vol. 12, Issue 4, pp. 3440-3442.

28. N.A. Bulychev, A.V. Ivanov. Study of Nanostructure of Polymer Adsorption Layers on the Particles Surface of Titanium Dioxide, International Journal of Nanotechnology, 2019, Vol. 16, Nos. 6/7/8/9/10, pp. $356-365$.

29. Formalev, V.F., Kartashov, É.M., Kolesnik, S.A. On the Dynamics of Motion and Reflection of Temperature Solitons in Wave Heat Transfer in Limited Regions // Journal of Engineering Physics and Thermophysics, 2020, 93(1), p. 10-15.

30. Formalev, V.F., Bulychev, N.A., Kuznetsova, E.L., Kolesnik, S.A. The Thermal State of a Packet of Cooled Microrocket Gas-Dynamic Lasers // Technical Physics Letters, 2020, 46(3), p. 245-248.

31. Formalev, V.F., Kolesnik, S.A., Kuznetsova, E.L., Rabinskiy, L.N. Origination and propagation of temperature solitons with wave heat transfer in the bounded area during additive technological processes // Periodico Tche Quimica. 2019. 16(33), p. 505-515.

32. Formalev, V.F., Kolesnik, S.A., Kuznetsova, E.L. Mathematical modeling of a new method of thermal protection based on the injection of special coolants // Periodico Tche Quimica. 2019.16(32), p. 598-607.

33. Rabinskiy, L.N., Tushavina, O.V. Composite Heat Shields in Intense Energy Fluxes with Diffusion// Russian Engineering Research, 2019, 39(9), p. 800-803.

34. Rabinskiy, L.N., Tushavina, O.V. Investigation of the influence of thermal and climate effects on the performance of tiled thermal protection of spacecraft//Periodico Tche Quimica, 2019, 16(33), p. 657-667.

35. Formalev, V.F., Kolesnik, S.A., Garibyan, B.A. Mathematical modeling of heat transfer in anisotropic plate with internal sinks // AIP Conference Proceedings, 2019, 2181, 020003.

36. Formalev, V.F., Kolesnik, S.A., Garibyan, B.A. Heat transfer with absorption in anisotropic thermal protection of high-temperature products // Herald of the Bauman Moscow State Technical University, Series Natural Sciences, 2019, (5), p. 35-49.

37. Formalev, V.F., Kolesnik, S.A., Garibyan, B.A. Analytical solution of the problem of conjugate heat transfer between a gasdynamic boundary layer and anisotropic strip //Herald of the Bauman Moscow State Technical University, Series Natural Sciences, 2020, 5(92), p. 44-59.

38. Vakhneev, S., Starovoitov, E. Damping of circular composite viscoelastic plate vibration under neutron irradiation// Journal of Applied Engineering Science, 2020, 18(4), p. 699-704. 